\title{
Multisensory Sensitivity is Related to Deep-Tissue but Not Cutaneous Pain Sensitivity in Healthy Individuals
}

This article was published in the following Dove Press journal: Journal of Pain Research

\author{
Dan Wang $\mathbb{D}^{\prime}$ \\ Shannon L Merkle (D) ${ }^{2}$ \\ Jennifer $E$ Lee $\mathbb{D}^{3,4}$ \\ Kathleen A Sluka (D) \\ Barbara Rakel (iD) \\ Thomas Graven-Nielsen (iD) ${ }^{5}$ \\ Laura A Frey-Law (D) ${ }^{\prime}$
}

'Department of Physical Therapy and Rehabilitation Science, Carver College of Medicine, University of lowa, lowa City, IA, USA; ${ }^{2}$ United States Army Research Institute of Environmental Medicine (USARIEM), Natick, MA, USA;

${ }^{3}$ Department of Psychology, Mount Mercy University, Cedar Rapids, IA, USA: ${ }^{4}$ College of Nursing, University of lowa, lowa City, IA, USA; ${ }^{5}$ Center for

Neuroplasticity and Pain (CNAP), SMI,

Aalborg University, Aalborg, Denmark
Correspondence: Laura A Frey-Law Department of Physical Therapy and Rehabilitation Science, Carver College of Medicine, University of lowa, I-252 Medical Education Bldg., 500 Newton Road, University of lowa, lowa City, IA 52242, USA

Tel + | 319-335-9804

$\mathrm{Fax}+1$ 319-335-9707

Email laura-freylaw@uiowa.edu
Purpose: Some individuals with chronic pain find daily life sensations (eg, noise, light, or touch) aversive. This amplification of multisensory sensations has been associated with centrally mediated plasticity; for example, greater multisensory sensitivity (MSS) occurs in patients with fibromyalgia than rheumatoid arthritis. However, whether MSS preferentially relates to pain measures which reflect central influences (eg, dynamic quantitative sensory testing (QST) or referred pain), or whether the MSS-pain relationship requires priming from chronic pain, is unknown. Thus, this cross-sectional study investigated the relationships between MSS assessed in a pain-free state and evoked pain sensitivity.

Methods: Experimental intramuscular infusion pain and multiple static and dynamic QST were assessed in 465 healthy, pain-free adults: pain thresholds using pressure (PPTs) and heat (HPTs), temporal summation of pain (TSP) using pressure, heat or punctate stimuli, and conditioned pain modulation (CPM) using pressure or heat test stimuli. MSS was assessed using 7 items from Barsky's Somatosensory Amplification Scale. Differences in pain and QST between sex-specific MSS quartiles were assessed, adjusting for multiple comparisons. All participants completed at least one intramuscular infusion condition, but not all were asked to complete each QST ( $\mathrm{n}=166-465)$.

Results: Both static and dynamic QST differed between highest and lowest MSS quartiles using pressure stimuli: lower PPTs (adjusted-p $<0.01$ ); increased pressure TSP (adjusted$\mathrm{p}=0.02$ ); lower pressure CPM (adjusted-p=0.01). However, none of the heat or punctate QST measures (HPTs, TSP, or CPM) differed between MSS quartiles (adjusted-p>0.05). Odds of experiencing TSP or referred pain was not greater, whereas CPM was 8-fold less likely, in those with highest MSS.

Conclusion: Normal variation in non-noxious MSS is related to both static and dynamic pain sensitivity, without sensitization associated with chronic pain, but is dependent on the QST stimulus. Thus, common influences on MSS and pain sensitivity may involve central mechanisms but are likely more complex than previously recognized.

Keywords: pain sensitivity, somatosensory amplification scale, experimental muscle pain, quantitative sensory testing, temporal summation of pain, conditioned pain modulation

\section{Plain Language Summary}

Heightened sensitivity to multiple senses, eg, bright lights, noises, strong perfumes, or clothing tags, is referred to as multisensory sensitivity (MSS) and has been observed in several chronic pain cohorts, particularly conditions thought to be driven by central mechanisms. These observations suggest that there may be a link between MSS and pain. However, whether this link only appears after adaptations that occur in the central nervous system in 
response to chronic pain, or whether it may be present even in pain-free adults was unknown. To test this, multiple pain sensitivity assessments were performed, including evoked measures of pressure, heat, punctate, and acidic muscle pain as well as a measure of MSS using items from an existing survey, the Somatosensory Amplification Scale, controlling for negative personality trait (ie, Neuroticism). We found that people with greater MSS tend to experience higher deep-tissue (eg, pressure) but not cutaneous (eg, heat) pain sensitivity. These findings indicate that the link between MSS and pain sensitivity does not require priming from chronic pain. Further, this relationship between pain sensitivity and MSS was not simple, but varied across test modalities. These results support the conclusion that elevated MSS can precede the development of chronic pain, and is not necessarily a consequence of chronic pain central nervous system adaptations. Thus, the MSS-pain relationship is more complex than previously recognized.

\section{Introduction}

Chronic pain affects over 100 million in the US alone. ${ }^{1}$ It is increasingly recognized that many clinical pain patients may involve combinations of nociceptive, nociplastic, and/ or neuropathic contributors. ${ }^{2-5}$ In particular, sensitization of central pain mechanisms, associated with nociplastic pain, likely contributes to the development and/or maintenance of chronic pain. ${ }^{4,5}$ Because central mechanisms and nociplastic changes cannot be assessed directly in humans, they are typically inferred as greater pain sensitivity using dynamic quantitative sensory testing (QST) relative to healthy individuals. ${ }^{4-8}$ More specifically, dynamic QST includes assessment of temporal summation of pain (TSP) and conditioned pain modulation (CPM). TSP refers to a wind-up response of $\mathrm{C}$ fibers to repetitive activation in animal models ${ }^{9}$ and has been well accepted as a psychophysical measure of net central facilitation of human pain in clinical settings. ${ }^{6,7}$ Similarly, CPM, which is psychophysically associated with diffuse noxious inhibitory controls, ${ }^{10}$ has been widely accepted as a measure of net endogenous descending inhibition of human pain in practice. 6,7

Emerging evidence suggests multisensory sensitivity (MSS) could potentially serve as an additional marker to reflect the status of CNS sensitivity as elevated MSS has been theorized to be attributable to a sensitized CNS. ${ }^{11-14}$ Indeed, a review showed pre-morbid assessments of high sensory sensitivity using QST were predictors of central sensitization. $^{14}$ Several pain populations, suspected to involve central mechanisms or nociplastic pain, report hypersensitivity to normal daily sensations, such as light touch, bright light or noise. For instance, patients with fibromyalgia reported greater MSS than a similar cohort of women with rheumatoid arthritis. ${ }^{12}$ Similarly, others report abnormally heightened sensory sensitivities (ie, phonophobia and photophobia) in people with fibromyalgia, ${ }^{13,15}$ complex regional pain syndrome, ${ }^{16}$ and migraine patients particularly during attacks ${ }^{17,18}$ compared to pain-free, control populations. These studies suggest that elevated MSS could be linked to chronic pain. However, it is unknown whether the link between MSS and pain develops in response to chronic pain, eg, due to nociplastic adaptations in the CNS, or may be present prior to the onset of a painful condition.

While elevated MSS assessed by various instruments has been observed in approximately 5-20\% of healthy, pain-free individuals, ${ }^{19,20}$ demonstrating normal variability, it is not clear the degree to which non-noxious MSS is related to various assessments of pain sensitivity in either healthy or clinical pain populations. For example, prior studies report mixed findings on the relationships between thermal pain sensitivity and non-noxious sensory sensitivity. $^{11,13,21}$ However, if MSS is related to chronic pain due to underlying shared central mechanisms as previously suggested, ${ }^{11,12,14}$ then one might expect MSS to be more closely related to dynamic QST and referred pain, both associated with central mechanisms, ${ }^{5-7,22}$ than to static QST or local pain. Unfortunately, studies are lacking that consider multiple QST modalities (eg, thermal and mechanical) using both static (thresholds) and dynamic (temporal summation or conditioned pain modulation) assessments or controlled experimentally induced pain models. In order to better understand the relevance of MSS in chronic pain, it is important to first determine the extent to which heightened multiple sensory sensitivity is related to pain sensitivity pre-morbidly. The use of a QST battery and experimental muscle pain in a healthy pain-free cohort provides an efficient way to investigate baseline associations between MSS and pain sensitivity, using clinically relevant measures while minimizing the potential confounding effects of patient-variation due to disease duration or underlying levels of pathology.

Accordingly, the primary purpose of this study was to assess the degree to which elevated MSS was associated with heightened pain sensitivity in previously pain-free adults, using a comprehensive battery of experimental muscle pain and QST measures. We hypothesized that individuals with heightened MSS would demonstrate 
greater dynamic than static QST pain sensitivity overall and greater risk of referred pain compared to those with low MSS. Our secondary aim was to evaluate for sex differences in these relationships, as women are greatly overrepresented in many chronic pain syndromes, ${ }^{23}$ and women typically show higher sensory sensitivity than men. ${ }^{11,24}$

\section{Materials and Methods}

\section{Participants}

All participants were recruited from the University and surrounding local community to participate in a study aimed at assessing predictors of muscle pain. A range of recruitment techniques were used, including posted flyers, ads in the University medical center daily lunch newsletter, an online listing of available studies, mass emails to University graduate students and staff, and word of mouth. Study inclusion criteria included: English-fluent, pain-free; ages from 18-55 years; and no history of chronic pain. Exclusion criteria included: significant medical conditions (eg, cardiac, asthma, rheumatologic, cancer, etc.); taking any medications other than birth control, allergy medications or multi-vitamins; significant selfreported psychiatric disorders; and history of significant musculoskeletal injuries, as previously described.$^{25}$ To be included in these analyses, additional inclusion criteria included completion of: at least one experimental muscle pain visit, the Somatosensory Amplification Scale (SSAS), ${ }^{26}$ and the Eysenck Personality QuestionnaireRevised (EPQ-R). ${ }^{27}$ Thus, 491 individuals were recruited for the overall muscle pain study with 465 meeting these additional analyses criteria. However, the specific sample sizes of QST pain sensitivity measures varied from 166 to 463 (Figure 1) as some QST assessments (eg, heat pain threshold (HPT), temporal summation of pain (TSP), conditioned pain modulation (CPM)) were added later in the study protocol.

\section{Ethics Approval}

This study was approved by the University of Iowa Biomedical Institutional Review Board (IRB approval \#200604757) for the protection of human subjects in compliance with federal human subjects regulations. Writteninformed consent prior to participation was obtained, as approved by the IRB. All participants were reimbursed for their time.

\section{Study Design}

This is secondary analyses of an observational, crosssectional study investigating factors associated with experimental muscle pain. Preliminary results are published elsewhere. ${ }^{25,28}$ Individuals participated in up to 3 visits to complete a series of surveys, exogenous anterior tibialis muscle pain, and QST pain sensitivity measures: pain thresholds, TSP and CPM, using pressure, heat and punctate stimuli. The surveys, the experimental muscle pain, and lower limb pressure pain thresholds (PPTs) were assessed in visits 1 and 2. A more comprehensive battery of QST was added as a third visit (see below), following a protocol revision part-way through the study. Accordingly, the sample size is largest for the experimental muscle pain and lower limb PPT outcomes and reduced for the remaining QST outcomes (Figure 1). General demographic information was collected as part of the surveys, including age, sex, height, weight, and general health. Body mass index (BMI) was computed from weight and height.

\section{Multisensory Sensitivity Assessment}

Non-noxious MSS was assessed using Barsky's Somatosensory Amplification Scale (SSAS) (Table 1). ${ }^{26}$ The SSAS assesses sensory amplification, or the tendency to perceive normal daily external or internal bodily sensations as being particularly unpleasant or bothersome, and has been used in various healthy and patient populations including pain patients. ${ }^{26,29-32}$ While this tool is sometimes used to assess symptom magnification, also referred to as hypochondriasis, ${ }^{32}$ it was originally described as assessing "perceived sensitivity to several unpleasant bodily sensations". ${ }^{26}$ The SSAS assesses multiple sensory systems, including somatic sensations, temperature, olfactory, and auditory sensitivity. Participants were asked to rate the degree to which each statement was true for them on a 5-point Likert scale, ranging from 1 ("not at all true") to 5 ("extremely true"). For example, items included: "Sudden loud noises really bother me," and "I hate to be too hot or too cold." While the original SSAS includes 10items, only 7 were summed for use as a measure of MSS (Table 1). Three items, "When someone else coughs, it makes me cough too;" "When I bruise myself, it stays noticeable for a long time;" and "I have a low tolerance for pain;" were assessed, but excluded from the current analysis as they are either less clearly relevant to MSS (eg, individuals may have physiologic reasons for bruising 


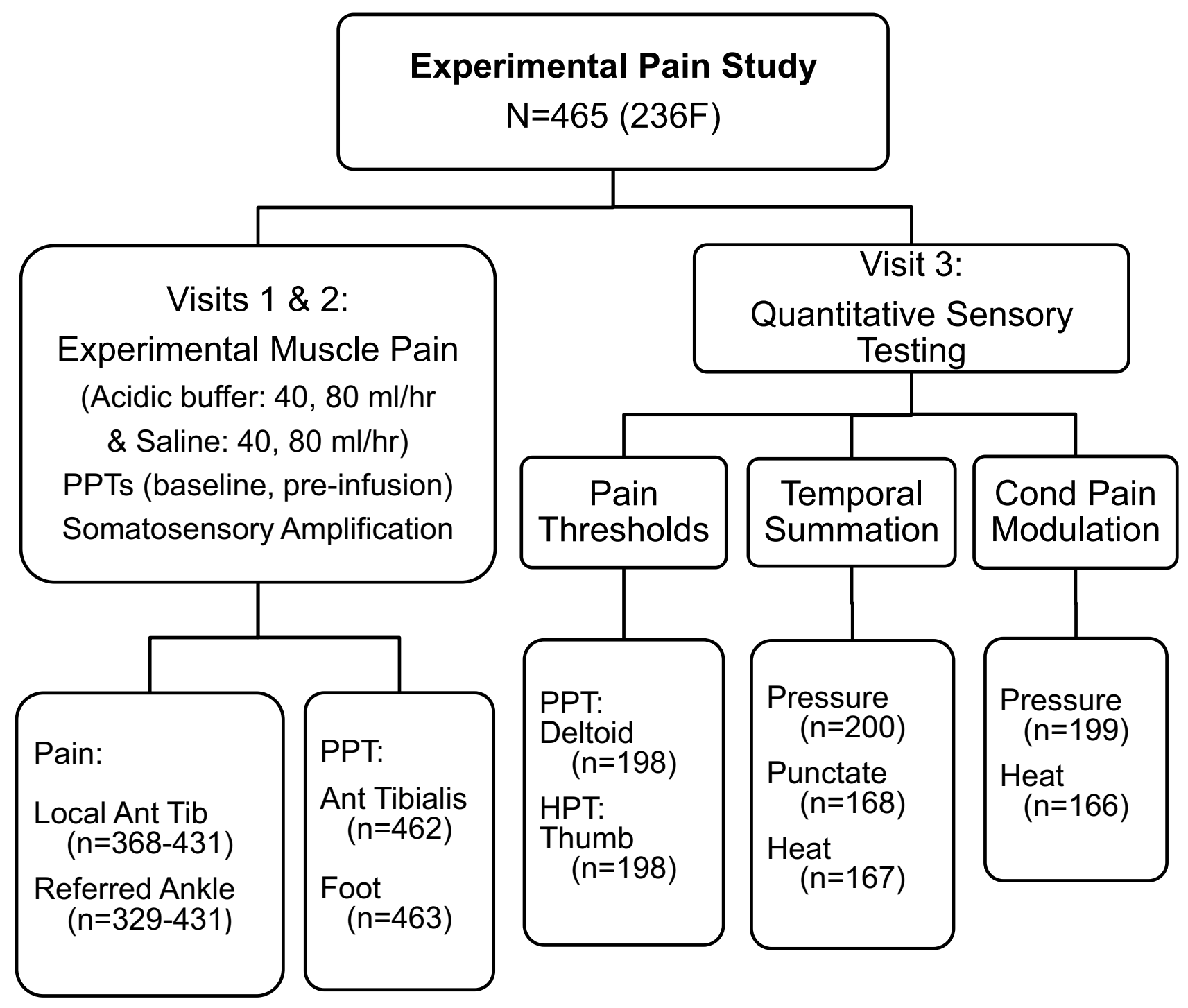

Figure I The schematic study design framework of the pain sensitivity assessments and associated sample sizes.

Abbreviations: PPT, pressure pain threshold; HPT, heat pain threshold; TSP, temporal summation of pain; CPM, conditioned pain modulation.

more easily, and coughing is not a sensory sensitivity) or it specifically assesses pain. We did not want to include a pain sensitivity item, and only include non-noxious MSS items, to minimize the risk of artificially elevating the associations between sensory sensitivity and pain sensitivity.

The original SSAS has been translated into multiple languages and has shown good internal consistency and test-retest repeatability. ${ }^{26,33,34}$ The sum of the 7-items had a total possible score ranging from 7 to 35 , where higher scores indicate higher non-noxious somatosensory amplification. Scores were categorized into sex-specific quartiles, as women typically report higher multisensory sensitivity than men, ${ }^{11,24}$ enabling high vs low somatosensory amplification comparisons while adjusting for sex differences. Using the current dataset, the 7-items resulted in McDonald's Omega $=0.79$, generally accepted as good internal consistency. ${ }^{35}$

To adjust for the potential confound of negative emotionality when assessing relationships between pain sensitivity and MSS, neuroticism was measured using the Eysenck Personality Questionnaire - Revised (EPQ-R), a standard personality instrument. ${ }^{27}$ The EQP-R Neuroticism subscale consists of 24 yes or no items that assess multiple aspects of negative emotionality. Higher scores indicate a personality type that is characterized as more anxious, fearful, and worrisome. The EPQ-R Neuroticism scale has demonstrated good reliability coefficients, with a range from 0.69 to 0.97 and a median of $0.83 .^{36}$ 
Table I Seven Items (Unshaded) from the 10-Item Somatosensory Amplification Scale (SSAS) Used to Assess Multisensory Sensitivity

\begin{tabular}{|l|}
\hline Individual Items of SSAS \\
\hline When someone else coughs, it makes me cough too. \\
\hline I cannot stand smoke, smog, or pollutants in the air. \\
\hline I am often aware of various things happening within my body. \\
\hline When I bruise myself, it stays noticeable for a long time. \\
\hline Sudden loud noises really bother me. \\
\hline I can sometimes hear my pulse or my heartbeat throbbing in my ear. \\
\hline I hate to be too hot or too cold. \\
\hline I am quick to sense the hunger contractions in my stomach. \\
\hline Even something minor, like an insect bite or a splinter, really bothers me. \\
\hline I cannot stand pain. \\
\hline
\end{tabular}

Notes: Grey highlighted items were not included in the non-noxious somatosensory amplification score for the current study. Reprinted from Journal of Psychiatric Research, 24(4), Barsky AJ, Wyshak G, Klerman GL, The somatosensory amplification scale and its relationship to hypochondriasis, 323-334, Copyright (1990), with permission from Elsevier. ${ }^{26}$

\section{Experimental Muscle Pain}

Intramuscular infusions into the mid-belly portion of the left anterior tibialis muscle were used to assess experimental muscle pain using two solutions at two infusion rates. Acidic ( $\mathrm{pH}$ 5.2) phosphate buffer (ie, acidic condition) or $0.9 \%$ isotonic saline (ie, saline condition) were infused at separate visits at both $40 \mathrm{~mL} / \mathrm{h}$ for $5 \mathrm{~min}$ and $80 \mathrm{~mL} / \mathrm{h}$ for 5 min with a two-minute washout period between rates, similar to previously reported methodology but with the addition of a faster rate. ${ }^{25}$ The study protocol initially used 20 and $40 \mathrm{~mL} / \mathrm{h}$ rates for $15 \mathrm{~min}$ each at separate visits, but due to low pain ratings was changed to 40 and $80 \mathrm{~mL} /$ $\mathrm{h}$ at a single visit for only $5 \mathrm{~min}$ each as peak pain occurred within 3-4 min. The order of the acidic vs saline infusions between visits, as well as the 40 versus 80 rate within each visit, was block randomized by assigned subject number, and balanced between men and women.

Anterior tibialis infusion models typically elicit mild to moderate muscle soreness locally at the infusion site (anterior shin) and referred pain at the anterior ankle joint. ${ }^{22,25}$ While local pain evoked by intramuscular infusion likely is a result of predominately peripheral hyperalgesia, referred pain is believed to be a centrally mediated phenomenon, a form of sensitized central pain mechanisms. ${ }^{5,22}$ Experimental muscle pain is rate dependent, ${ }^{25,37}$ thus the faster rate $(80 \mathrm{~mL} / \mathrm{hr})$ conditions were expected to induce greater pain than the slower rate ( $40 \mathrm{~mL} / \mathrm{hr}$ ). Further, the buffered acidic condition is typically more painful than normal saline as it is thought to activate acid sensing ion channels, ${ }^{25,38}$ whereas the saline condition only induces mechanical distension. Thus, the four conditions provide varying levels of intramuscular painful stimuli, from very low (saline $40 \mathrm{~mL} / \mathrm{h}$ ) to moderate (acidic $80 \mathrm{~mL} / \mathrm{h}$ ) pain. This provides a means to assess muscle pain sensitivity across a range of controlled stimuli.

Pain ratings were verbally assessed every $30 \mathrm{~s}$ during the infusions using a verbal $0-10$ scale (Borg CR10 scale), ${ }^{39}$ where 0 represented "no pain at all" and 10 signified "the maximum pain they have ever imagined". Participants could rate their pain with fractions or decimals if they chose. Participants were asked to rate both their local pain ("at the infusion site") and referred pain ("at the ankle"). The peak pain ratings for the local and referred pain sites were extracted for each condition. The Borg CR10 scale has been shown to be a valid instrument to measure pain intensity. ${ }^{39}$

\section{Pressure and Heat Pain Thresholds}

Pressure pain thresholds (PPTs) were measured using a digital, hand-held pressure algometer (Somedic AB, Farsta Sweden) with a standard $1 \mathrm{~cm}^{2}$ rubber-tipped probe at a rate of $30 \mathrm{kPa} / \mathrm{s}$. Participants were instructed to press a hand-held trigger when they first felt pain from the pressure ("approximately 1 out of 10 pain"). Only baseline PPTs (prior to the intramuscular infusion) were considered in the current analyses. Multiple locations were assessed: the anterior tibialis muscle belly, averaging measures from just above and below the infusion site; the web space between the 1st and 2nd metatarsals on the ipsilateral foot, and the middle deltoid muscle belly of the left arm (assessed during the third QST visit, see Figure 1). The average of four baseline repetitions was used as the PPT value for the anterior tibialis and foot; two pre- and two post-catheter insertions as they were not significantly different across time. The average of three repetitions was used for the deltoid PPT location. Subjects were familiarized with the PPT assessment using one practice round at each site prior to data collection.

Heat pain thresholds (HPTs) were measured on the left thenar eminence of the hand (thumb) using a computercontrolled TSA-II Neurosensory Analyzer (Medoc II, Israel) and a $16 \times 16 \mathrm{~mm}$ Peltier thermode to determine cutaneous thermal pain sensitivity. The temperature was 
increased at a rate of $0.5^{\circ} \mathrm{C} / \mathrm{s}$ from a baseline temperature of $39^{\circ} \mathrm{C}$ to a maximum temperature of $52.5^{\circ} \mathrm{C}$. Participants were instructed to press a computer mouse button when they first felt pain from the heat stimulus ("approximately 1 out of 10 pain"). HPTs were computed as the average of three trials with $5 \mathrm{~s}$ intervals between each, following one practice trial for familiarization.

\section{Pressure, Heat, and Punctate Temporal Summation of Pain}

Temporal summation of pain (TSP) was assessed as the human analog to wind-up, to indirectly assess CNS facilitation of pain. ${ }^{9,40}$ Numerous evidence supports that TSP can be induced if the frequency of nociceptive stimuli is equal to or greater than $0.33 \mathrm{~Hz}{ }^{41-45}$ TSP was assessed using three modalities: pressure, heat, and punctate stimuli. Each stimulus was applied at a frequency of 0.5 $\mathrm{Hz}^{46-49}$ for 30 seconds (ie, 15 repetitions). Participants were instructed to rate their pain intensity initially (after 2 repetitions), again mid-way (15 seconds), after the final stimulation (30 seconds) and any after sensations (45 seconds and 60 seconds) using the 0-10 pain scale. ${ }^{39}$ TSP was computed as the peak pain minus the initial pain, where positive values (ie, increased pain) represents presence of TSP.

To assess pressure TSP, a custom device was used to apply a deep-tissue mechanical stimulus with a $1 \mathrm{~cm}^{2}$ rubber tip over the extensor carpi radialis brevis muscle on the left dorsal forearm. The pressure stimulus (ie, force applied) was individually determined for each participant targeting pain ratings of 3 out of 10 , assessed prior to starting the TSP test. Heat TSP was assessed using repetitive-phasic heat stimuli applied on the volar side of the left forearm using the $16 \times 16 \mathrm{~mm}$ Medoc TSA-II probe. The pre-programmed heat TSP protocol varied the temperature from a baseline of $41^{\circ} \mathrm{C}$ to the destination temperature of $49^{\circ} \mathrm{C}$ at a rate of $10^{\circ} \mathrm{C} / \mathrm{s}$. Punctate TSP was performed using a $300 \mathrm{~g}$ von Frey filament applied to the volar side of the left wrist. The same rate (1 sec on: $1 \mathrm{sec}$ off), number of stimuli ( $\mathrm{n}=15)$ and pain assessments $(0-10$ pain scale) were used for each TSP procedure.

\section{Pressure and Heat Conditioned Pain Modulation}

Conditioned pain modulation (CPM) is the human analog to diffuse noxious inhibitory controls (DNIC), an indirect measure of descending pain inhibition. ${ }^{50}$ It was assessed as the reduction in pain sensitivity to a test stimulus at a remote site following a conditioning pain stimulus. Pressure CPM was assessed using the mean of 3 PPTs of the left deltoid muscle as the test stimuli; heat CPM was assessed using the mean of 3 HPTs at the left thenar eminence as the test stimuli (same upper extremity locations used for PPTs and HPTs described above). For both pressure and heat CPM, the conditioning stimulus was right hand immersion to the wrist in circulating $0-2{ }^{\circ} \mathrm{C}$ water for $1 \mathrm{~min}$ (or as tolerated). CPM was computed as the difference in mean pain thresholds (immediately post - pre-conditioning values). A positive value (increased pain threshold) indicated the presence of CPM (ie, centrally mediated pain inhibition). A minimum 5 min washout interval separated the two CPM tests to allow subjects to recover from the prior cold-water hand immersion. The order of QSTs was block randomized between modalities within each class of QST (ie, heat vs pressure or punctate) but thresholds were always tested first, followed by TSP, and finally CPM to minimize the likelihood that the more intense cold-water immersion influenced threshold or summation responses.

\section{Statistical Analyses}

All values are presented in the text as mean \pm standard deviation (SD), medians and interquartile ranges (IQR), or proportions unless otherwise noted. MSS sex-specific quartiles were computed; each participant was assigned to one sex-specific quartile classification, where 1st quartile represented the lowest and 4th quartile represented the highest sensory sensitivity groupings. This was done to enable comparisons between groups of individuals to be performed based on their MSS levels. Demographic variables were compared between sexes and across MSS quartiles using analysis of variance (ANOVA) or ChiSquare $\left(X^{2}\right)$ tests as appropriate. All statistical analyses were performed using SAS 9.4 (SAS Institute, USA) with significance set at $\mathrm{p}<0.05$.

Differences in pain sensitivity outcomes (dependent variables) were assessed between those in the highest three MSS quartiles relative to the reference (1st) quartile (independent variable) using generalized linear models (GLM). To test whether the MSS-pain sensitivity relationships differed between sexes, an omnibus test including an interaction term, ie, sex by MSS quartile, was initially performed. When there were no significant sex interactions, the GLMs were then repeated without the interaction term to optimize study power. All analyses were adjusted for a priori determined confounders (age, sex, BMI, and neuroticism). Model 
residuals were tested for normality; when violated, Box Cox transformations of the study variables were applied as necessary to appropriately meet normality assumptions. Outliers were identified using Q-Q plots and removed. Specifically, data points that were clearly separated from the reference line of the Q-Q plot were identified as outliers and removed from the data analyses. The GLM analyses were then repeated to ensure the residuals met normality assumptions. ${ }^{51}$ The false discovery rate (FDR) was used to adjust p-values for multiple comparisons.

Standardized effect sizes (Cohen's d and 95th CI) were computed for each pain sensitivity outcome between the 4th and 1st sex-specific MSS quartiles using transformed data as appropriate. Cohen's d values were computed from adjusted least square (LS) means and SD using an online calculator (https://www.psychometrica.de/effect_size. html). Adjustment for unequal sample sizes was performed. $^{52}$ Effect sizes were operationally defined using the following cut-off values for small $(\mathrm{d} \geq 0.2)$, medium $(d \geq 0.5)$, and large $(d \geq 0.8)$ effect sizes. ${ }^{53}$

In addition to quartile comparisons, which assess for differences relative to a reference group, we used multiple regression to assess the linear nature of the relationship between continuous pain sensitivity outcomes (dependent variables) and MSS scores (independent variable). Again, the transformed pain sensitivity variables identified for the GLM analyses were used for the multiple regression, with age, sex, BMI, and neuroticism included as model covariates. Model coefficients of determination $\left(\mathrm{R}^{2}\right)$, beta coefficients for MSS and associated p-values were assessed. Lastly, multiple logistic regression analyses were used to assess the odds of experiencing dichotomous pain sensitivity outcomes: presence of referred pain, presence of TSP, and absence of CPM for those in the highest versus the lowest MSS quartiles (4th versus 1st). This was assessed to evaluate whether MSS is associated with the presence/absence of central pain processing markers, as referred pain, TSP and CPM are described as indirect markers of a sensitized CNS., ${ }^{4,5}$

\section{Results}

\section{Participant Demographics, MSS and Pain Sensitivity}

Overall, 491 participants were recruited; 465 met the inclusion criteria for these analyses. See Table 2 for summary demographic information of subjects included in data analyses. Overall, MSS scores were higher in women $(19.1 \pm 4.3)$ than men $(17.5 \pm 4.0, \mathrm{p}=0.0001)$, resulting in the approximately 1.5-point higher cut-off values for women across each quartile (see supporting materials Table S1). Age and neuroticism differed across sex-specific MSS quartiles $(\mathrm{p} \leq 0.01)$, but no differences in sex distribution, race, or BMI were observed (Table 2). People in the highest MSS quartile (the most sensitive to non-noxious sensory inputs) were significantly younger and had higher neuroticism scores than those in the lowest quartile $(\mathrm{p} \leq 0.01)$. Demographic information on subjects excluded from the analyses are presented in Table S2 and descriptive statistics for the observed cohorts and those with missing data are presented in Table S3.

Women showed higher pressure pain sensitivity (lower PPTs) than men at the anterior tibialis and deltoid sites (adjusted-p $<0.01$, see supporting materials Table S4). Men reported slightly higher local muscle pain from the least noxious infusion condition, saline at $40 \mathrm{~mL} / \mathrm{hr}$ (adjusted-p $=0.04$, Table S4). Whereas, despite similar local pain intensities, women were more likely to

Table 2 Characteristics of Study Participants Overall and by MSS Sex-Specific Quartiles

\begin{tabular}{|c|c|c|c|c|c|c|c|}
\hline & \multicolumn{2}{|l|}{ All $(\mathrm{N}=465)$} & \multicolumn{4}{|c|}{ MSS Sex-Specific Quartiles } & \multirow{2}{*}{$\begin{array}{l}\text { Quartile } \\
\text { P-value }\end{array}$} \\
\hline & Mean (SD) or N (\%) & Range & QI $(n=\mid 29)$ & Q2 (n=127) & Q3 $(n=107)$ & Q4 $(n=102)$ & \\
\hline Age & $26.2(7.7)$ & $18-54$ & $28.1^{a, b, c}(9.0)$ & $26.0^{\mathrm{a}, \mathrm{b}, \mathrm{c}}(7.6)$ & $25.6^{\mathrm{b}, \mathrm{c}}(6.7)$ & $24.9^{\mathrm{b}, \mathrm{c}}(6.8)$ & 0.01 \\
\hline BMI $\left(\mathrm{kg} / \mathrm{m}^{2}\right)$ & $24.6(3.8)$ & $|7-4| .3$ & $25.1^{\mathrm{a}}(4.3)$ & $24.4^{\mathrm{a}}(3.5)$ & $24.8^{\mathrm{a}}(4.0)$ & $24.0^{\mathrm{a}}(3.2)$ & 0.17 \\
\hline Female & $236(50.8)$ & - & $60(46.5)$ & $77(60.6)$ & $50(46.7)$ & $49(48.0)$ & 0.08 \\
\hline Caucasian & 404 (86.9) & - & $105(81.4)$ & 116 (9I.3) & 94 (87.9) & $89(87.3)$ & 0.39 \\
\hline 7-item SSAS & $18.3(4.2)$ & $9-30$ & $13.3^{\mathrm{a}}(1.9)$ & $17.4^{\mathrm{b}}(1.0)$ & $19.9^{c}(1.2)$ & $24.1^{d}(2.3)$ & $<0.0001$ \\
\hline EPQ-R Neuroticism & $9.1(5.1)$ & $0-23$ & $7.3^{\mathrm{a}}(5.0)$ & $8.8^{\mathrm{b}}(5.0)$ & $9.6^{\mathrm{b}, \mathrm{c}}(4.6)$ & $11.3^{\mathrm{d}}(5.1)$ & $<0.0001$ \\
\hline
\end{tabular}

Notes: Values with same upper letter (a, b, c, d) indicated no difference with each other. False discovery rate was used for multiple comparisons between MSS sex-specific quartiles (4th = highest sensory sensitivity; Ist = lowest sensory sensitivity). Mean (SD) or N (\%) were presented if appropriate. The significant values for the ANOVA model were highlighted in bold ( $\mathrm{p} \leq 0.05)$.

Abbreviations: MSS, multisensory sensitivity; SSAS, somatosensory amplification scale; EPQ-R Neuroticism, Eysenck Personality Questionnaire-Revised Neuroticism. 
experience referred pain than men from the acidic infusions: odds ratios $1.7[1.1,2.6]$ and $2.1[1.2,3.7]$ for 40 and $80 \mathrm{~mL} / \mathrm{hr}$, respectively (see supporting materials, Table S5). No other pain sensitivity measures differed significantly between men and women.

There were no significant sex interactions with sexspecific MSS quartiles for all but two pain sensitivity outcomes: local muscle pain from saline infusion $(80 \mathrm{~mL} / \mathrm{hr})$ and PPTs at the deltoid. Thus, for these two outcomes only, the models were analyzed with the interaction term in addition to adjusting for sex, BMI, age and neuroticism as covariates.

\section{Experimental Muscle Pain}

Local muscle pain intensity was greater for those in the highest (4th) than those in the lowest (1st) sex-specific MSS quartiles in only one of the four conditions: acidic infusion at $40 \mathrm{~mL} / \mathrm{hr}(\mathrm{d}=0.43$; adjusted- $\mathrm{p}=0.01$, Figure $2 \mathrm{~A}$ and supporting materials, Table S6). Local muscle pain from the $80 \mathrm{~mL} / \mathrm{hr}$ saline infusion showed different MSSmuscle pain relationships between men and women (significant sex interaction, $p=0.01$ ). Follow-up testing revealed women but not men showed significant differences in muscle pain between MSS quartiles ( $p=0.05, d=0.56$, Table S6), after adjusting for multiple comparisons (Figure $3 \mathrm{~A})$. However, there were significant linear trends $(\mathrm{p}<$ 0.03 ) between MSS and experimental muscle pain for two conditions: saline and acidic infusions at $40 \mathrm{~mL} / \mathrm{hr}$.

Referred pain intensity was not compared across MSS quartiles using general linear modeling, as approximately $21.6-54.5 \%$ of individuals did not report any referred pain across infusion conditions (see supporting materials Table

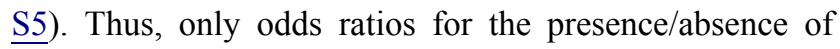
referred pain were assessed. However, the odds of experiencing referred pain during any of the infusion conditions was not significantly different between the extreme MSS quartiles (4th vs 1st; Figure 4).

\section{Pain Thresholds}

PPTs were significantly lower (higher pain sensitivity; adjusted-p < 0.007) in individuals in the two highest MSS quartiles at the anterior tibialis muscle $(\mathrm{d}=0.59$ and 0.54), and in highest MSS quartile at the foot $(\mathrm{d}=$ 0.43; Figure 2B and Table S6) compared to those in the lowest MSS quartile. However, at the deltoid, men and women showed different MSS - PPT relationships (ie, significant sex interaction with $\mathrm{p}=0.04$ ) with follow-up testing revealing that neither men or women showed significant differences in PPTs between MSS quartiles after adjusting for multiple comparisons (Figure 3B). Conversely, HPTs did not differ between MSS quartiles (adjusted-p $=0.71$; Figure 2C). Similar findings were observed when evaluating for linear trends between pain thresholds and MSS (supporting materials, Table S6) where only PPTs at the lower limbs significantly varied with MSS scores.

\section{Temporal Summation of Pain}

Pressure TSP was significantly greater in those in the highest compared to the lowest sex-specific MSS quartiles (adjusted-p $=0.03, \mathrm{~d}=0.57$, Figure 2D and supporting materials, Table S6). However, neither heat nor punctate TSP differed significantly between MSS quartiles ( $p>$ 0.35; Figure 2D and Table S6). Similarly, the linear trend analyses produced consistent results - only pressure TSP varied with MSS (Table S6). When assessing simply for the absolute presence/absence of TSP between MSS extreme quartiles using logistic regression, no significant differences were noted. However, the odds of experiencing punctate TSP for the highest vs lowest MSS quartiles approached significance: 2.36 [0.84, 6.65] (Figure 4).

\section{Conditioned Pain Modulation}

Pressure CPM was lower (ie, less pain inhibition) in those with the highest (4th quartile) versus those with the lowest (1st quartile) MSS scores ( $d=0.67$; adjusted-p $=0.01$ Figure 2E and supporting materials, Table S6). Again, however, no differences in heat CPM were observed between the highest versus the lowest somatosensory quartiles (Figure 2F and Table S6). Further, the trend analyses produced similar results - only pressure CPM varied with MSS (Table S6). Finally, people with greater MSS (2nd, $3 \mathrm{rd}$, and 4th quartiles) were nearly three to eight times less likely to experience pressure CPM than those in the lowest (1st) quartile (Figure 4 and Table S7).

\section{Discussion}

This is the first study to examine the relationships between MSS and multiple measures of pain sensitivity. While previous research has shown heightened sensitivity to nonnoxious stimuli in several chronic pain conditions, our results indicate self-reported MSS in the pain-free state is related to select QST measures without adapted neuroplastic changes from pre-existing pain conditions. Further, MSS is uniquely related to pressure QST assessments. No cutaneous (heat or punctate) pain sensitivity assessments were related to self- 

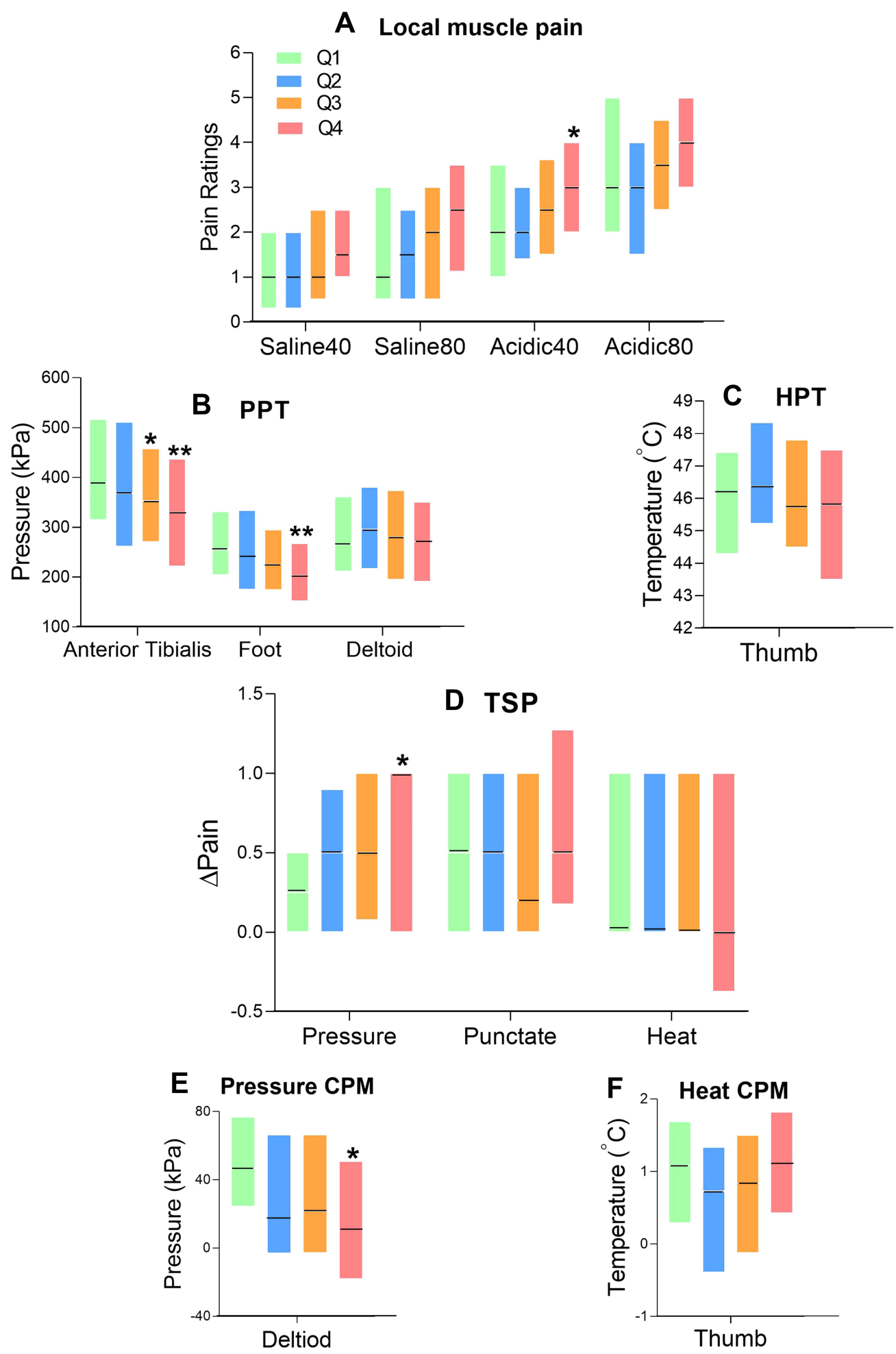

Figure 2 Boxplots showing median (black bars) and 25th-75th percentiles (IQR boxes) of each pain sensitivity outcome by sex-specific MSS quartiles: (A) local pain ratings from 4 intramuscular infusion conditions; (B) pressure pain thresholds (PPT) at 3 sites; (C) heat pain thresholds (HPT); (D) temporal summation of pain (TSP) using 3 different stimuli; (E) condition pain modulation using PPTs as the test stimulus (pressure CPM); and (F) CPM using HPTs as the test stimulus (heat CPM). Significant differences between MSS quartiles (eg, 4th $=$ highest sensory sensitivity) and the referent MSS quartile (Ist, lowest) are indicated $\left(* * p \leq 0.01 *^{*} \leq 0.05\right)$ based on transformed data but graphs show original data for clarity. 

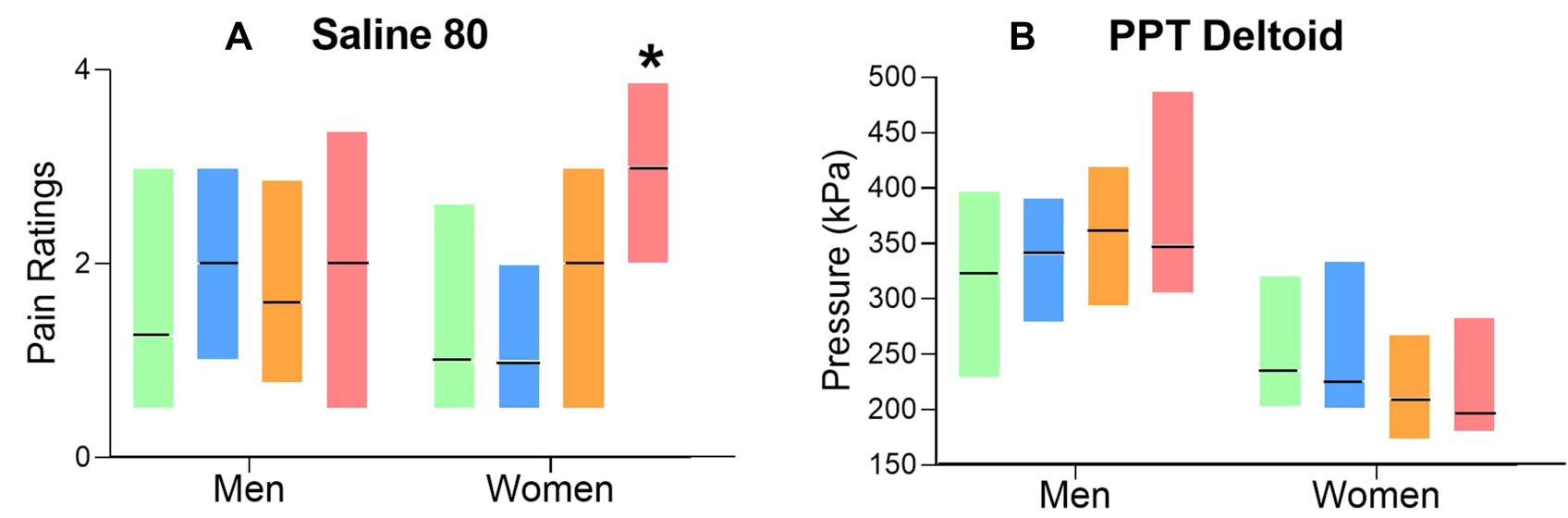

Figure 3 Boxplots showing median (black bars) and 25th-75th percentiles (IQR boxes) of (A) local pain ratings between men and women from the saline infusion at $80 \mathrm{~mL}$ $\mathrm{hr}$ and (B) pressure pain thresholds (PPT) at the deltoid muscle are shown by sex-specific MSS quartiles. Significant sex-MSS interactions were observed for both conditions; yet the follow-up stratified analyses by sex only show the significant difference on saline infusion at $80 \mathrm{~mL} / \mathrm{hr}$ in women between MSS quartiles (eg, 4 th $=$ highest sensory sensitivity) and the referent MSS quartile (Ist, lowest) $\left({ }^{*} \mathrm{p} \leq 0.05\right)$.

\section{Odds Ratios}

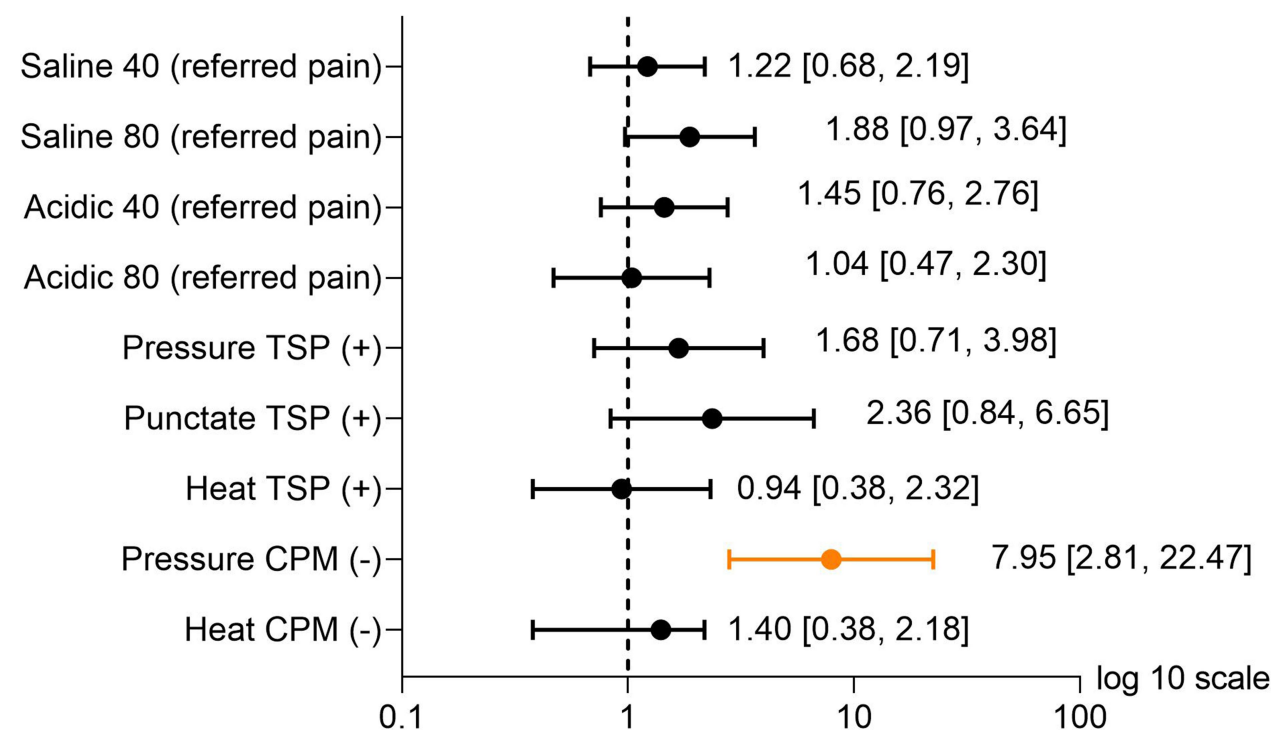

Figure 4 Odds ratios (ORs) and their associated $95 \%$ confidence intervals $(\mathrm{Cl})$ for experiencing: referred pain from the 4 intramuscular infusions, the presence of temporal summation (TSP (+)), and the absence of conditioned pain modulation (CPM (-)) to each stimulus in people with high (4th quartile) versus low (Ist quartile) MSS. All ORs were adjusted for age, sex, BMI, and neuroticism. Orange symbols and lines highlight significant ORs $(p \leq 0.05)$.

reported MSS, despite temperature tolerance being one of the items used to assess MSS. These results may suggest heightened MSS may serve as a risk factor for deep-tissue pain sensitivity. Contrary to our initial hypotheses, these relationships were not clearly differentiated between static and dynamic QST and did not vary substantially between men and women. Thus, common influences on MSS and pain sensitivity may involve central mechanisms but are likely more complex than previously recognized.

\section{Dynamic versus Static QST}

There is a general consensus that dynamic QST provides indirect indicators of central pain processing, ${ }^{6,7,54,55}$ while static QST, such as pain thresholds, may involve a mix of peripheral and central components. Dynamic and static QST have been widely applied to measure pain sensitivity in clinical pain populations and to further infer the underlying mechanisms. ${ }^{6,8,56-58}$ For instance, PPT and TSP to mechanical stimuli have been predictive of factors 
associated with the fear-avoidance model, such as pain catastrophizing and pain-related disability. ${ }^{8}$ This supports the application of QST and its relevance to diverse aspects of the pain experience. As TSP and CPM are widely accepted as measures reflecting central facilitation and inhibition of pain, ${ }^{6}$ the associations observed in our study may indicate that noxious and non-noxious sensory processing may be linked centrally, even prior to the onset of a clinical pain syndrome. However, we cannot rule out that peripheral nervous system sensitivity may also play a role in shaping the varying degrees of associations observed between MSS and pain sensitivity, based on the associations also observed with PPTs. Thus, more complex mechanisms than previously appreciated may underlie the integration, modulation, and processing of painful and non-painful sensory inputs.

When interpreting observed differences in QST, consideration should be made regarding the underlying measurement error, using criteria such as twice the standard error of measurement (SEM). ${ }^{59}$ For example, two PPTs and the pressure CPM were significantly different between those in the highest versus the lowest MSS quartiles. Prior research has reported a SEM for PPTs as 20.6 $\mathrm{kPa}^{59}$ We observed PPT differences (Q4 versus Q1) of 59.4 and $81.8 \mathrm{kPa}$ for the foot and leg locations which exceed this $2 * \mathrm{SEM}$ criteria (ie, $41.2 \mathrm{kPa}$ ). This suggests that in addition to being statistically meaningful, these PPT difference may be interpreted as real, measurable differences between the two extreme MSS groups. However, the pressure CPM difference (Q4 versus Q1) was only $34.3 \mathrm{kPa}$, which while larger than the SEM does not exceed $2 *$ SEM. Instead, many investigators simply characterize the absence or presence of CPM rather than its magnitude. The 8-fold decrease in pressure CPM incidence in MSS Q4 versus Q1 reveals the high risk of developing compromised central inhibition to pain in highly sensitive people.

\section{Deep-Tissue versus Cutaneous Pain Sensitivity}

The distinction between deep- and superficial-pain sensitivity and MSS was not anticipated, as there is little prior work for comparison. One prior study with a small sample of healthy adults $(n=30)$ also observed no relationship between heat CPM and sensory over-responsiveness, but conversely found higher heat pain ratings in those with greater over-responsiveness to sensory inputs. ${ }^{21}$ While the previous study only partially supports our findings, our results suggest that MSS is differentially related to QST based more on the stimulus modality than the type of test performed.

Deep-tissue and cutaneous pain processing exhibit several unique features that may contribute to or be associated with this finding. For example, while both muscle and cutaneous afferents project to similar lamina layers in dorsal horn, ${ }^{60,61}$ muscle afferents demonstrate greater probability of glutamate release and higher expression of AMPA receptors than cutaneous afferents. ${ }^{61}$ Additionally, tonic inhibition of sensory inputs to dorsal horn neurons is stronger for deep than cutaneous mechanical stimuli, indicating differential descending modulation between deeptissue and cutaneous signals. ${ }^{62}$ Clinically, deep-tissue pain is perceived differently, often diffuse and described as aching and cramping, while cutaneous pain is well localized and frequently described as sharp, stabbing or burning. ${ }^{63-65}$ Further, deep, but not cutaneous, pain has been associated with autonomic symptoms. ${ }^{64,66}$ While it is not clear which, if any, of these differences may be relevant to the unique shared variance observed between deeptissue QST and MSS, the observed relationships suggest assessment of MSS in musculoskeletal pain conditions may have clinical value.

\section{Potential Shared Brain Regions}

While identifying the exact CNS pathways or regions responsible for this shared relationship between MSS and pain sensitivity is beyond the scope of this study, multiple candidate neurophysiological substrates have been identified. Brain regions involved in both painful and nonpainful aversive stimuli processing include: thalamus, middle and posterior cingulate cortices, anterior insula, dorsal striatum, dorsomedial prefrontal cortex, posterior cingulate cortex, hippocampus, and midbrain areas. ${ }^{67-70}$ More specifically, the thalamus is involved in modifying noxious and non-noxious sensory signals, with integration of, and communication between, multiple sensory pathways. ${ }^{71}$ Interestingly, all senses except olfaction directly transmit sensory information from the thalamus to the cerebral cortex. ${ }^{72,73}$ Structures in the limbic system, such as the insula, cingulate and medial prefrontal cortex, respond to both noxious inputs and a wide range of other sensory and affective events. ${ }^{74-76}$ A subset of the rostral ventromedial medulla neurons, known as pain-modulating neurons, not only respond to noxious heat but also bright lights, providing another potential convergence site for 
noxious and non-noxious signal processing. ${ }^{68}$ However, this does not readily match the lack of association observed between heat pain sensitivity and somatosensory amplification in the current study, nor similar results found involving heat TSP or CPM in individuals with and without elevated multisensory sensitivity. ${ }^{13,21}$ Whereas, healthy individuals with greater sensory sensitivity reported higher pain ratings to noxious stimuli than those without. $^{21}$ Accordingly, the underlying mechanisms explaining the potential shared variance between noxious and non-noxious sensitivity remain unclear.

\section{MSS as a Potential Risk Factor for Chronic Pain}

Prior studies have demonstrated associations between elevated sensory sensitivity and pain in select clinical conditions. $^{11,15}$ Similarly, higher proportions of individuals with fibromyalgia, ${ }^{12,77}$ migraine, ${ }^{78}$ and complex regional pain syndrome ${ }^{16}$ exhibit heightened MSS compared to healthy adults. Bar-Shalita suggests this evidence supports the role of MSS as a risk factor. ${ }^{16}$ Yet, these studies are unable to differentiate whether the experience of pain sensitized the CNS, thereby producing amplified sensory perception, or whether multisensory hypersensitivity precedes the development of the pain condition. Certainly, the relationships between pain and sensory sensitivity may differ when the CNS is in a sensitized state. However, our findings that highly sensitive healthy individuals experience heightened deep-tissue pain sensitivity support the possibility that MSS may be a risk factor for chronic pain. While relatively little is known of the risk profile associated with heightened MSS, patients characterized with amplified sensory sensitivity, such as autism spectrum disorder, have also been identified as having compromised endogenous pain modulation. ${ }^{79,80}$ Future longitudinal studies are needed to clarify the role of MSS as a chronic pain risk factor.

\section{Potential for Response Bias}

Self-reported MSS and self-reported pain could be related simply in part due to reporting biases or due to elevated negative affect contributing to a heightened perceived negativity overall. However, our results do not support this possibility. The relationship between MSS and deep-tissue pain sensitivity was not simply explained by neuroticism, a personality trait that is characterized by negative emotions such as anxiety, guilt, fear and depression. ${ }^{81}$ Indeed, neuroticism is correlated with selfreported sensory processing sensitivity, ${ }^{24,82}$ pain sensitivity, ${ }^{83}$ and we observed higher neuroticism scores in people with the highest MSS scores. However, the muscle and deep-tissue mechanical pain sensitivity assessments remained significantly related to MSS even after adjustment for neuroticism, indicating that negative emotionality is not the driving factor in this relationship. This finding is consistent with previous findings that self-reported sensory sensitivity remained strongly associated with greater activation in brain areas involved in high-order visual processing after controlling for neuroticism and introversion. ${ }^{84}$ Despite several differences in methodology, including the tool to assess sensory sensitivity and the means of assessing non-noxious stimuli processing, our studies collectively support that these findings are not simply due to confounding from disproportional negative emotionality. Further, the significant relationships observed between MSS and select QST did not only occur based on subjective pain ratings, but also by indicating differences in stimulus intensity threshold (ie, in $\mathrm{kPa}$ or degree units) to produce pain thresholds and CPM. This, along with the unique findings that selfreported MSS only predicted pressure-based QST but no other cutaneous forms of QST, further substantiates that reporting bias is not a likely explanation.

\section{Study Limitations}

Several limitations of this study are worth noting as they may limit generalizability. First, our population was predominantly Caucasian. Second, the sample sizes varied across QST assessments, such that the deltoid vs lower extremity PPT differences may be due to differences in study power. However, the larger effect sizes for deeptissue versus cutaneous QST regardless of sample size suggest these results are robust. Additionally, subjects who volunteer to do pain studies may be less pain sensitive or fearful than the general population, yet our study still observes a significant relationship between increased sensory sensitivity and higher deep-tissue pain sensitivity. Lastly, MSS was assessed using 7-items from the SSAS, a self-report instrument that does not involve assessments of taste, light or tactile sensitivity which are common sensations in daily life. However, currently, there is no gold-standard instrument for measuring MSS that cover all primary senses, particularly a brief and freely available instrument focusing on sensory sensitivity and not confounded by other 
constructs such as coping strategies or avoidance behaviors. Future studies to evaluate available MSS tools and potentially develop improved MSS metrics are warranted.

\section{Conclusions}

Overall, this study uniquely demonstrates that MSS assessed in a pain-free state is related to both static and dynamic deep-tissue QST, indicating this relationship does not require the presence of chronic pain. Whether more generalized and magnified associations could also occur across modalities when in a more sensitized CNS state, as may occur with chronic pain, remains unknown. Our study provides a baseline reference highlighting a link between MSS and pain sensitivity that may serve as a comparison to future assessments involving chronic pain and the contributions of MSS to the pain experience. Further, the parallel relationships between MSS with static and dynamic QST, but only with pressure stimuli, observed in our study suggests the MSS-pain interaction involves more complex mechanisms which may not be limited to central components. Given the intrinsic MSS-pain associations demonstrated in our findings, with the higher prevalence of elevated MSS in select chronic pain conditions, the link between MSS and pain sensitivity may play a larger role in the development or maintenance of chronic pain than previously recognized. Finally, this study investigating pain-free populations paves the way for further research in this important area of predicting chronic pain, particularly central sensitization or nociplastic pain.

\section{Disclaimer}

The content and views contained herein are solely the responsibility of the authors and are not to be construed as official or reflecting the views of the University of Iowa, the US Department of the Army, the US Department of Defense, the National Institutes of Health, the International Association for the Study of Pain (IASP), or the Danish National Research Foundation. The results of this study do not constitute endorsement by any of the above-mentioned entities. The results of the study are presented clearly, honestly, and without fabrication, falsification, or inappropriate data manipulation.

\section{Supporting Materials}

Additional supporting materials (Tables S1 - S7) can be found online.

\section{Abbreviations}

MSS, Multisensory Sensitivity; QST, Quantitative Sensory Testing; PPT, Pressure Pain Threshold; HPT, Heat Pain Threshold; TSP, Temporal Summation of Pain; CPM, Conditioned Pain Modulation, SSAS, Somatosensory Amplification Survey; EPQ-R, Eysenck Personality Questionnaire - Revised; CNS, Central Nervous System.

\section{Acknowledgments}

The authors would like to thank Mr. Chris DeBlois and Ms. Jackie Loesche for their assistance with data collection. This research was supported in part by funding from the National Institutes of Health NIAMS grant \#s: K01AR056134 and R03AR065197; the International Association for the Study of Pain (IASP) Collaborative Research Award, and University of Iowa Clinical \& Translational Science Award UL1TR002537 (REDCap) from the National Center for Advancing Translational Sciences; and the US Army (student support for SLM). The Center for Neuroplasticity and Pain (CNAP) is supported by the Danish National Research Foundation (DNRF121).

\section{Author Contributions}

All authors made substantial contributions to conception and design, acquisition of data, or analysis and interpretation of data; took part in drafting the article or revising it critically for important intellectual content; agreed to submit to the current journal; gave final approval of the version to be published; and agree to be accountable for all aspects of the work.

\section{Funding}

This research was supported in part by funding from the National Institutes of Health NIAMS grant \#s: K01AR056134 and R03AR065197; the International Association for the Study of Pain (IASP) Collaborative Research Award, and University of Iowa Clinical \& Translational Science Award UL1TR002537 (REDCap) from the National Center for Advancing Translational Sciences; and the US Army (student support for SLM). The Center for Neuroplasticity and Pain (CNAP) is supported by the Danish National Research Foundation (DNRF121).

\section{Disclosure}

Dr. Merkle received support for her doctoral training as a member of the US Army during the conduct of this study. 
Dr Sluka serves as a consultant for Novartis Consumer Healthcare/GSK Consumer Healthcare, receives a research grant from Pfizer Inc., and receives royalties from IASP Press, and reports personal fees from IASP Press, personal fees from GSK Consumer Health, personal fees from Psychogenics, outside the submitted work. Dr. GravenNielsen reports grants from Danish National Research Foundation, outside the submitted work. Dr. Frey-Law reports grants from NIH, grants from IASP, UL1TR002537 (for REDCap use) from NIH (ICTS), student sponsorship from DoD, during the conduct of the study; grants from NIH, outside the submitted work. The aforementioned authors report no other potential conflicts of interest for this work. The remaining authors have no conflicts of interest to declare.

\section{References}

1. Simon LS. Relieving pain in America: a blueprint for transforming prevention, care, education, and research. J Pain Palliat Care Pharmacother. 2012;26(2):197-198. doi:10.3109/15360288.2012.678473

2. Kosek E, Cohen M, Baron R, et al. Do we need a third mechanistic descriptor for chronic pain states? Pain. 2016;157(7):1382-1386. doi:10.1097/j.pain.0000000000000507

3. Freynhagen R, Parada HA, Calderon-Ospina CA, et al. Current understanding of the mixed pain concept: a brief narrative review. Curr Med Res Opin. 2019;35(6):1011-1018. doi:10.1080/ 03007995.2018.1552042

4. Latremoliere A, Woolf CJ. Central sensitization: a generator of pain hypersensitivity by central neural plasticity. J Pain. 2009;10 (9):895-926. doi:10.1016/j.jpain.2009.06.012

5. Woolf CJ. Central sensitization: implications for the diagnosis and treatment of pain. Pain. 2011;152(3 Suppl):S2-15. doi:10.1016/j. pain.2010.09.030

6. Uddin Z, MacDermid JC. Quantitative sensory testing in chronic musculoskeletal pain. Pain Med. 2016;17(9):1694-1703. doi:10.109 3/pm/pnv105

7. Mackey IG, Dixon EA, Johnson K, Kong JT. Dynamic quantitative sensory testing to characterize central pain processing. J Vis Exp. 2017;120.

8. Uddin Z, Woznowski-Vu A, Flegg D, Aternali A, Wickens R, Wideman TH. Evaluating the novel added value of neurophysiological pain sensitivity within the fear-avoidance model of pain. Eur J Pain. 2019;23(5):957-972. doi:10.1002/ejp.1364

9. Mendell LM. Physiological properties of unmyelinated fiber projection to the spinal cord. Exp Neurol. 1966;16(3):316-332. doi:10.1016/0014-4886(66)90068-9

10. Le Bars D, Dickenson AH, Besson JM. Diffuse noxious inhibitory controls (DNIC). I. Effects on dorsal horn convergent neurones in the rat. Pain. 1979;6(3):283-304. doi:10.1016/0304-3959(79)90049-6

11. Dixon EA, Benham G, Sturgeon JA, Mackey S, Johnson KA, Younger J. Development of the Sensory Hypersensitivity Scale (SHS): a self-report tool for assessing sensitivity to sensory stimuli. J Behav Med. 2016;39(3):537-550.

12. Wilbarger JL, Cook DB. Multisensory hypersensitivity in women with fibromyalgia: implications for well being and intervention. Arch Phys Med Rehabil. 2011;92(4):653-656. doi:10.1016/j.apmr.2010.10.029

13. Bar-Shalita T, Vatine JJ, Yarnitsky D, Parush S, Weissman-Fogel I. Atypical central pain processing in sensory modulation disorder: absence of temporal summation and higher after-sensation. Exp Brain Res. 2014;232(2):587-595. doi:10.1007/s00221-013-3767-y
14. Clark J, Nijs J, Yeowell G, Goodwin PC. What are the predictors of altered central pain modulation in chronic musculoskeletal pain populations? A systematic review. Pain Physician. 2017;20(6):487-500.

15. Geisser ME, Glass JM, Rajcevska LD, et al. A psychophysical study of auditory and pressure sensitivity in patients with fibromyalgia and healthy controls. J Pain. 2008;9(5):417-422. doi:10.1016/j. jpain.2007.12.006

16. Bar-Shalita T, Livshitz A, Levin-Meltz Y, Rand D, Deutsch L, Vatine JJ. Sensory modulation dysfunction is associated with complex regional pain syndrome. PLoS One. 2018;13(8):e0201354. doi:10.1371/journal.pone.0201354

17. Main A, Dowson A, Gross M. Photophobia and phonophobia in migraineurs between attacks. Headache. 1997;37(8):492-495. doi:10.1046/j.1526-4610.1997.3708492.x

18. Vingen JV, Pareja JA, Støren O, White LR, Stovner LJ. Phonophobia in migraine. Cephalalgia. 1998;18(5):243-249. doi:10.1046/j.14682982.1998.1805243.x

19. Ahn RR, Miller LJ, Milberger S, McIntosh DN. Prevalence of parents' perceptions of sensory processing disorders among kindergarten children. Am J Occup Ther. 2004;58(3):287-293. doi:10.5014/ajot.58.3.287

20. Wilbarger P, Wilbarger JL. Sensory Defensiveness in Children Aged 2-12: An Intervention Guide for Parents and Other Caretakers. Therapro; 1991.

21. Weissman-Fogel I, Granovsky Y, Bar-Shalita T. Sensory over-responsiveness among healthy subjects is associated with a pronociceptive state. Pain Pract. 2018;18(4):473-486.

22. Graven-Nielsen T. Fundamentals of muscle pain, referred pain, and deep tissue hyperalgesia. Scand J Rheumatol Suppl. 2006;122:1-43.

23. Mogil JS. Sex differences in pain and pain inhibition: multiple explanations of a controversial phenomenon. Nat Rev Neurosci. 2012;13(12):859-866.

24. Aron EN, Aron A. Sensory-processing sensitivity and its relation to introversion and emotionality. J Pers Soc Psychol. 1997;73 (2):345-368. doi:10.1037/0022-3514.73.2.345

25. Frey Law LA, Sluka KA, McMullen T, Lee J, Arendt-Nielsen L, Graven-Nielsen T. Acidic buffer induced muscle pain evokes referred pain and mechanical hyperalgesia in humans. Pain. 2008;140 (2):254-264. doi:10.1016/j.pain.2008.08.014

26. Barsky AJ, Wyshak G, Klerman GL. The somatosensory amplification scale and its relationship to hypochondriasis. J Psychiatr Res. 1990;24(4):323-334. doi:10.1016/0022-3956(90)90004-A

27. Eysenck HJ, Eysenck SBG. Manual of the Eysenck Personality Scales (EPS Adult): Comprising the EPQ-Revised (EPQ-R), EPQ-R Short Scale, Impulsiveness (IVE) Questionnaire. Hodder \& Stoughton; 1991.

28. Merkle SL Exploring pain \& movement relationships: is greater physical activity associated with reduced pain sensitivity \& does endogenous muscle pain alter protective reflexes in the upper extremity? 2016.

29. Le Borgne M, Boudoukha AH, Petit A, Roquelaure Y. Chronic low back pain and the transdiagnostic process: how do cognitive and emotional dysregulations contribute to the intensity of risk factors and pain? Scand J Pain. 2017;17:309-315. doi:10.1016/j.sjpain.2017.08.008

30. Schroeder S, Gerlach AL, Martin A. Implicit affective evaluation of somatosensory sensations in patients with noncardiac chest pain. J Behav Ther Exp Psychiatry. 2014;45(3):381-388. doi:10.1016/j. jbtep.2014.04.002

31. Çakmak S, Özbek HT, Işı AG, et al. The relationship between somatic sense perception levels and comorbid psychiatric diseases in chronic pain patients. Agri. 2019;31(4):183-194.

32. Nakao M, Barsky AJ. Clinical application of somatosensory amplification in psychosomatic medicine. Biopsychosoc Med. 2007;1:17.

33. Aghayousefi A, Oraki M, Mohammadi N, Farzad V, Daghaghzadeh H. Reliability and validity of the Farsi version of the somatosensory amplification scale. Iran J Psychiatr Behav Sci. 2015;9(3):e233. doi:10.17795/ijpbs-233 
34. Bridou M, Aguerre C. Validity of the French form of the somatosensory amplification scale in a non-clinical sample. Health Psychol Res. 2013;1(1):e11. doi:10.4081/hpr.2013.668

35. Hayes AF, Coutts JJ. Use omega rather than Cronbach's alpha for estimating reliability. Commun Methods Meas. 2020;14(1):1-24. doi:10.1080/19312458.2020.1718629

36. Caruso JC, Witkiewitz K, Belcourt-Dittloff A, Gottlieb JD. Reliability of scores from the eysenck personality questionnaire: a reliability generalization study. Educ Psychol Meas. 2001;61 (4):675-689. doi:10.1177/00131640121971437

37. Issberner U, Reeh PW, Steen KH. Pain due to tissue acidosis: a mechanism for inflammatory and ischemic myalgia? Neurosci Lett. 1996;208(3):191-194. doi:10.1016/0304-3940(96)12576-3

38. Immke DC, McCleskey EW. Lactate enhances the acid-sensing $\mathrm{Na}+$ channel on ischemia-sensing neurons. Nat Neurosci. 2001;4 (9):869-870. doi:10.1038/nn0901-869

39. Borg G. Borg's Perceived Exertion and Pain Scales. Champaign, IL, US: Human Kinetics; 1998.

40. Dickenson AH, Sullivan AF. Evidence for a role of the NMDA receptor in the frequency dependent potentiation of deep rat dorsal horn nociceptive neurones following $\mathrm{C}$ fibre stimulation. Neuropharmacology. 1987;26(8):1235-1238. doi:10.1016/00283908(87)90275-9

41. Staud R, Robinson ME, Price DD. Temporal summation of second pain and its maintenance are useful for characterizing widespread central sensitization of fibromyalgia patients. J Pain. 2007;8 (11):893-901. doi:10.1016/j.jpain.2007.06.006

42. Cuellar JM, Montesano PX, Antognini JF, Carstens E. Application of nucleus pulposus to L5 dorsal root ganglion in rats enhances nociceptive dorsal horn neuronal windup. J Neurophysiol. 2005;94 (1):35-48.

43. Price DD, Hu JW, Dubner R, Gracely RH. Peripheral suppression of first pain and central summation of second pain evoked by noxious heat pulses. Pain. 1977;3(1):57-68. doi:10.1016/0304-3959(77) 90035-5

44. Whitsel B, Tommerdahl M, Kohn A, Vierck C, Favorov O Pain Imaging, Progress in Pain Research and Management. 2000.

45. Price DD. Characteristics of second pain and flexion reflexes indicative of prolonged central summation. Exp Neurol. 1972;37 (2):371-387. doi:10.1016/0014-4886(72)90081-7

46. Kong JT, Bagarinao E, Olshen RA, Mackey S. Novel characterization of thermal temporal summation response by analysis of continuous pain vs time curves and exploratory modeling. J Pain Res. 2019;12:3231-3244. doi:10.2147/JPR.S212137

47. Horn-Hofmann C, Kunz M, Madden M, Schnabel E-L, Lautenbacher S Interactive effects of conditioned pain modulation and temporal summation of pain — the role of stimulus modality. PAIN. 2018;159(12):2641-2648. doi:10.1097/j.pain.0000000000001376

48. Li J, Simone DA, Larson AA. Windup leads to characteristics of central sensitization. Pain. 1999;79(1):75-82. doi:10.1016/S03043959(98)00154-7

49. Petersen KK, Jensen MB, Graven-Nielsen T, Hauerslev LV, ArendtNielsen L, Rathleff MS. Pain catastrophizing, self-reported disability and temporal summation of pain predict self-reported pain in low back pain patients 12 weeks after general practitioner consultation a prospective cohort study. Clin J Pain. 2020.

50. Willer JC, Roby A, Le Bars D. Psychophysical and electrophysiological approaches to the pain-relieving effects of heterotopic nociceptive stimuli. Brain. 1984;107(4):1095-1112. doi:10.1093/brain/ 107.4.1095

51. Q-Q Plot (Quantile to Quantile Plot). In: The Concise Encyclopedia of Statistics. Springer, New York, NY. https://doi.org/10.1007/978-0387-32833-1_331

52. Hedges LV, Olkin I. Statistical Methods for Meta-Analysis. Academic press; 2014.
53. Cohen J. Statistical power analysis. Curr Dir Psychol Sci. 1992;1 (3):98-101. doi:10.1111/1467-8721.ep10768783

54. Williams DA. Phenotypic features of central sensitization. J Appl Biobehav Res. 2018;23(2):e12135. doi:10.1111/jabr.12135

55. Treede RD. The role of quantitative sensory testing in the prediction of chronic pain. Pain. 2019;160(Suppl 1):S66-s69. doi:10.1097/j. pain.0000000000001544

56. Uddin Z, Macdermid J, Packham T. Ice-water (cold stress) immersion testing. J Physiother. 2013;59(4):277. doi:10.1016/S1836-9553(13) 70211-X

57. Uddin Z, MacDermid J, Packham T. Clinical implementation of two quantitative sensory tests: cold stress test and the ten test. Physiother Pract Res. 2014;35:33-40. doi:10.3233/PPR-130030

58. Uddin Z, MacDermid JC, Galea V, Gross AR, Pierrynowski MR. The current perception threshold test differentiates categories of mechanical neck disorder. J Orthop Sports Phys Ther. 2014;44(7):532-540, c531. doi:10.2519/jospt.2014.4691

59. Kennedy DL, Kemp HI, Wu C, Ridout DA, Rice ASC. Determining real change in conditioned pain modulation: a repeated measures study in healthy volunteers. J Pain. 2019.

60. Cramer GD, Darby SA. Clinical Anatomy of the Spine, Spinal Cord, and ANS-E-Book. Elsevier Health Sciences; 2017.

61. Li J, Baccei ML. Functional organization of cutaneous and muscle afferent synapses onto immature spinal lamina i projection neurons. $J$ Neurosci. 2017;37(6):1505-1517. doi:10.1523/JNEUROSCI.3164-16.2016

62. Yu XM, Mense S. Response properties and descending control of rat dorsal horn neurons with deep receptive fields. Neuroscience. 1990;39(3):823-831. doi:10.1016/0306-4522(90)90265-6

63. Kellgren J. Observations on referred pain arising from muscle. Clin Sci. 1938;3(176):1937-1938.

64. Mense S. Nociception from skeletal muscle in relation to clinical muscle pain. Pain. 1993;54(3):241-289. doi:10.1016/0304-3959(93) 90027-M

65. Torebjörk HE, Ochoa JL, Schady W. Referred pain from intraneural stimulation of muscle fascicles in the median nerve. Pain. 1984;18 (2):145-156. doi:10.1016/0304-3959(84)90882-0

66. Feinstein B, Langton JN, Jameson RM, Schiller F. Experiments on pain referred from deep somatic tissues. J Bone Joint Surg Am. 1954;36(5):981-997. doi:10.2106/00004623-195436050-00007

67. Hayes DJ, Northoff G. Common brain activations for painful and non-painful aversive stimuli. BMC Neurosci. 2012;13:60.

68. Martenson ME, Halawa OI, Tonsfeldt KJ, et al. A possible neural mechanism for photosensitivity in chronic pain. Pain. 2016;157 (4):868-878. doi:10.1097/j.pain.0000000000000450

69. Rolls ET. Limbic systems for emotion and for memory, but no single limbic system. Cortex. 2015;62:119-157. doi:10.1016/j.cortex.2013.12.005

70. Tyll S, Budinger E, Noesselt T. Thalamic influences on multisensory integration. Commun Integr Biol. 2011;4(4):378-381. doi:10.4161/ cib. 15222

71. Noseda R, Constandil L, Bourgeais L, Chalus M, Villanueva L. Changes of meningeal excitability mediated by corticotrigeminal networks: a link for the endogenous modulation of migraine pain. J Neurosci. 2010;30(43):14420-14429. doi:10.1523/ JNEUROSCI.3025-10.2010

72. Haberly LB, Price JL. The axonal projection patterns of the mitral and tufted cells of the olfactory bulb in the rat. Brain Res. 1977;129 (1):152-157. doi:10.1016/0006-8993(77)90978-7

73. Price JL, Powell TP. Certain observations on the olfactory pathway. J Anat. 1971;110(Pt 1):105-126.

74. Lopez-Sola M, Pujol J, Wager TD, et al. Altered functional magnetic resonance imaging responses to nonpainful sensory stimulation in fibromyalgia patients. Arthritis Rheum. 2014;66(11):3200-3209. doi:10.1002/art.38781

75. Lopez-Sola M, Woo CW, Pujol J, et al. Towards a neurophysiological signature for fibromyalgia. Pain. 2017;158(1):34-47. doi:10.1097/j. pain.0000000000000707 
76. Sharvit G, Corradi-Dell'Acqua C, Vuilleumier P. Modality-specific effects of aversive expectancy in the anterior insula and medial prefrontal cortex. Pain. 2018;159(8):1529-1542. doi:10.1097/j. pain.0000000000001237

77. Watson NF, Buchwald D, Goldberg J, Noonan C, Ellenbogen RG. Neurologic signs and symptoms in fibromyalgia. Arthritis Rheum. 2009;60(9):2839-2844. doi:10.1002/art.24772

78. Granovsky Y, Shor M, Shifrin A, Sprecher E, Yarnitsky D, BarShalita T. Assessment of responsiveness to everyday non-noxious stimuli in pain-free migraineurs with versus without aura. J Pain. 2018;19(8):943-951. doi:10.1016/j.jpain.2018.03.008

79. Duerden EG, Taylor MJ, Lee M, McGrath PA, Davis KD, Roberts SW. Decreased sensitivity to thermal stimuli in adolescents with autism spectrum disorder: relation to symptomatology and cognitive ability. J Pain. 2015;16(5):463-471. doi:10.1016/j.jpain.20 15.02 .001
80. Gu X, Zhou TJ, Anagnostou E, et al. Heightened brain response to pain anticipation in high-functioning adults with autism spectrum disorder. Eur J Neurosci. 2018;47(6):592-601. doi:10.1111/ejn.13598

81. Costa Jr PT, McCrae RR. Four ways five factors are basic. Pers Individ Dif. 1992;13(6):653-665. doi:10.1016/0191-8869(92)90236-I

82. Listou Grimen H, Diseth $\AA$. Sensory processing sensitivity: factors of the highly sensitive person scale and their relationships to personality and subjective health complaints. Percept Mot Skills. 2016;123 (3):637-653. doi:10.1177/0031512516666114

83. Lee JE, Watson D, Frey Law LA. Lower-order pain-related constructs are more predictive of cold pressor pain ratings than higher-order personality traits. $J$ Pain. 2010;11(7):681-691. doi:10.1016/j.jpain.2009.10.013

84. Jagiellowicz J, Xu X, Aron A, et al. The trait of sensory processing sensitivity and neural responses to changes in visual scenes. Soc Cogn Affect Neurosci. 2011;6(1):38-47.

\section{Publish your work in this journal}

The Journal of Pain Research is an international, peer reviewed, open access, online journal that welcomes laboratory and clinical findings in the fields of pain research and the prevention and management of pain. Original research, reviews, symposium reports, hypothesis formation and commentaries are all considered for publication. The manuscript management system is completely online and includes a very quick and fair peer-review system, which is all easy to use. Visit http:// www.dovepress.com/testimonials.php to read real quotes from published authors. 